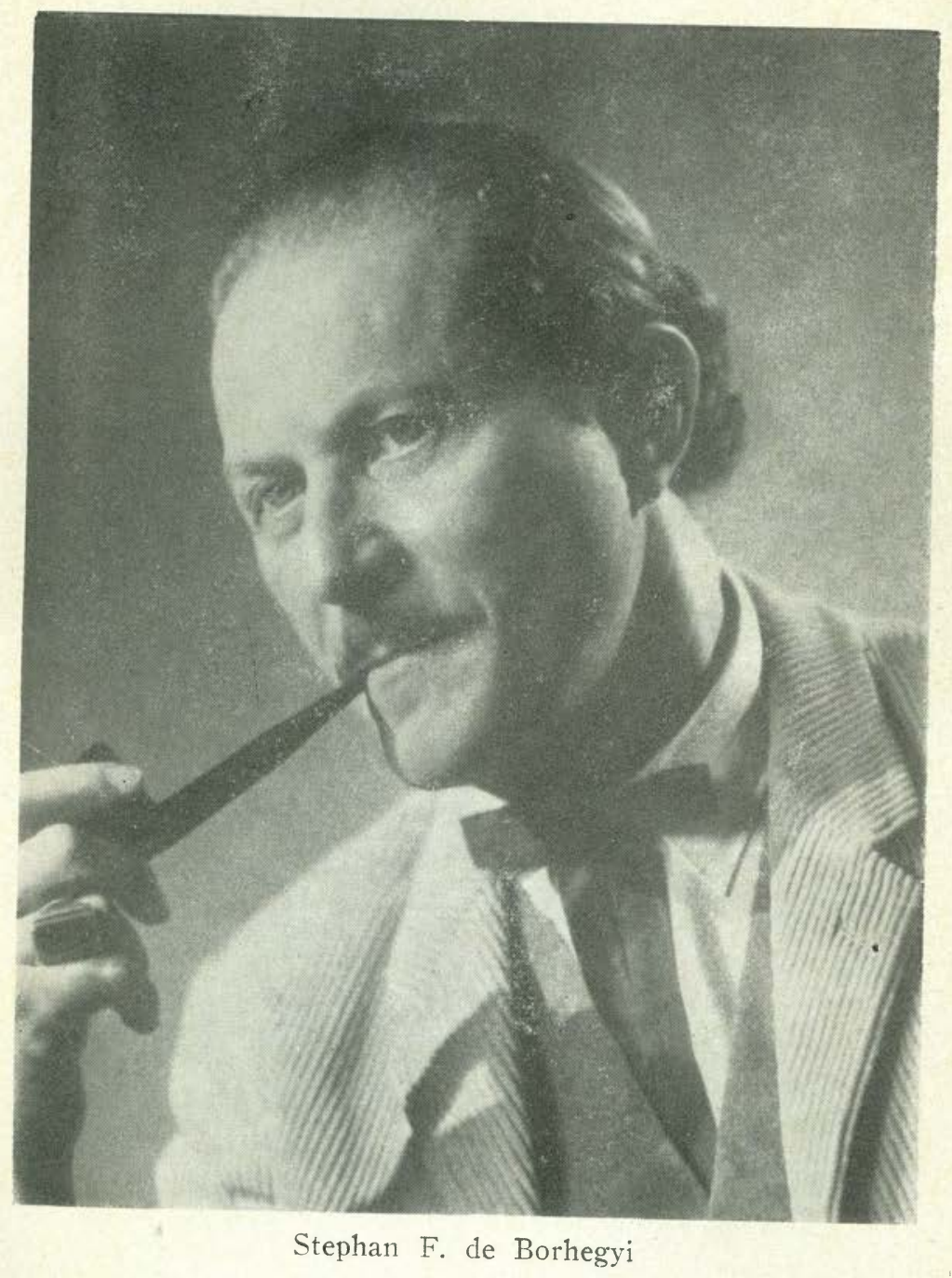

Estudios de Cultura Maya. Vol. VIII, 1970

Facultad de Filosofía y Letras/Coordinación de Humanidades

Centro de Estudios Mayas, UNAM

http://www.iifilologicas.unam.mx/estculmaya/ 


\section{STEPHAN F. DE BORHEGYI}

(1921-1969)

Por Carlos Navarrete

Instituto de Investigaciones Históricas. UNAM.

En la actualidad, cuando los arqueólogos han dejado de ser personajes de aventuras en expediciones fantásticas, la personalidad de Stephan Borhegyi parecía desmentir esa afirmación, pues gustaba de mantener la leyenda del explorador incansable y reflejar, al mismo tiempo, las cualidades del investigador moderno, al que las circunstancias colocan en el centro de una intensa actividad social.

Este equilibrio - a veces sutil, otras desafiantes-, se reflejaba en sus modales y actitudes, en su pronunciación deliberadamente balcánica, en el estilo personal de su ropa, y en la disposición para disfrutar, ya la incomodidad y los sencillos alimentos en un campamento o el goce de la mejor mesa europea; la capacidad para adentrarse en la naturaleza, sin menoscabo de la desenvoltura necesaria en los grandes salones de sociedad.

Nativo de Hungría, tuvo desde niño la oportunidad de recorrer Europa y de familiarizarse con el ambiente de los museos y los monumentos antiguos, que lo llevaron a estudiar historia clásica y medieval. La Segunda Guerra Mundial interrumpió sus estudios y lo vio vestir el uniforme militar con el grado de oficial. Pasada la conflagración, se graduó en la Universidad Peter Pazmani de Budapest.

Fue nombrado conservador asistente de arqueología clásica en el Museo Nacional de Hungría hasta 1949, cuando viajó a los Estados Unidos con una beca de la Fundación Viking, para realizar estudios de arqueología y etnología de América en la Universidad de Arizona. Tuvo la suerte de conocer y relacionarse con el doctor Alfred Kidder, quien lo invitó a trabajar en Guatemala donde realizaba investigaciones arqueológicas.

Este viaje, patrocinado por la Fundación Bollingen, coincidió con el movimiento revolucionario guatemalteco que, entre otros objetivos de transformación cultural, había fundado el Instituto Nacional de Antropología. Steve fue el organizador de las colecciones del nuevo 
museo, donde catalogó más de un millón de piezas, creando para ello un sistema de clasificación que, en su momento, se consideró de los más prácticos y avanzados del mundo.

Fueron años definitivos en su carrera de investigador. El conocimiento directo de los materiales guatemaltecos y su contacto diario con algunos de los mejores especialistas en el área maya, lo situaron para siempre en el terreno de la arqueología mesoamericana. Aún recuerdo los elogiosos comentarios de la prensa y la larga serie de discursos efímeros, que suscitó la entrega del material clasificado al gobierno de Guatemala.

A su regreso a los Estados Unidos realizó estudios de postgraduado en la Universidad de Yale. De 1954 a 1959, fue director del Museo Stovall de la Universidad de Oklahoma donde, a su vez, impartió cursos de antropología. De allí pasó con el mismo cargo al museo de Milwaukee, donde volvió a mostrar sus cualidades de organizador; su actividad fue de tal impulso que en pocos años hizo crecer el museo, hasta convertirlo en uno de los más importantes de los Estados Unidos. Fundó comités para allegar fondos a la institución, encabezó programas de conferencias, ideó nuevos sistemas de exhibición, y mantuve "su" museo en intercambio constante de experiencias con otros de América y Europa.

Quizá fuera esa gran disposición para ser siempre la cabeza en cualquier tarea de carácter organizativo y su tremenda actividad en muchos campos, lo que impidió que se manifestara completamente su calidad de investigador. Porque su obra escrita, aunque numerosa e importante, siempre se limitó a un tipo de ensayos y resúmenes, de monografías y notas, a los que les faltó tiempo y mayor dedicación para ser definitivos. Fue un magnífico presentador de materiales, publicó acertadas observaciones sobre aspectos tipológicos y, sobre todo, tuvo imaginación para intentar conclusiones e insinuar caminos a la investigación. Pero fue muy presuroso para redondear y concluir lo que impulsaba.

Tuve el placer de trabajar a su lado durante la temporada de exploraciones en Chinkultic, Chiapas, en los primeros meses de 1969. Fue un tiempo sumamente agradable y feliz para quienes trabajamos en ese lugar, pues el paisaje, la tranquilidad de la estación y la cortesía de los habitantes se adentraron en nosotros, haciéndonos desear una permanencia eterna en ese rincón, que era casi un refugio.

Una tarde le pregunté si le gustaría pasar la vida en un lugar así y morir tranquilo y reposado; me contestó: "No. Vivir afuera, para retornar de vez en cuando. $Y$ en cuanto a morir tranquilo, no me 
convence. Yo soy de los que no pueden morir de viejos en la cama."

Así fue. El 26 de septiembre de 1969, Steve falleció en un accidente de tráfico.

\section{BIBLIOGRAFÍA}

MESOAMÉRICA: (ARQUEOLOGÍA Y ETNOLOGÍA)

1. "Tlaloc Effigy Jar from the Guatemalan National Museum", Notes on Middle American Archaeology and Ethnology, Carnegie Institution of Washington, Department of Archaeology, vol. Iv, No. 96, pp. 55-59. Cambridge, Mass., 1950.

2. "Rim-Head Vessels and Cone-Shaped Effigy Prongs of the PreClassic Period at Kaminaljuyú, Guatemala", Notes on Middle American Archaeology and Ethnology, Carnegie Institution of Washington, Department of Archaeology, vol. IV, No. 97, pp. 60-80. Cambridge, Massachusetts, 1950.

3. "A Group of Jointed Figurines in the Guatemalan National Museum", Notes on Middle American Archaeology and Ethnology, Carnegie Institution of Washington, Department of Archaeology, vol. Iv, No. 100, pp. 93-99. Cambridge, Massachusetts, 1950.

4. "Estudio Arqueológico en la Falda Norte del Volcán de Agua", Revista del Instituto de Antropología e Historia de Guatemala, vol. II, No. 1, pp. 3-22. Guatemala, 1950.

5. "Notas Sobre Sellos de Barro Existentes en el Museo Nacional de Arqueología y Etnología de Guatemala", Revista del Instituto de Antropología e Historia de Guatemala, vol. II, No. 2, pp. 16-26. Guatemala, 1950.

6. "A Study of Three-Pronged Incense Burners from Guatemala and Adjacent Areas", Notes on Middle American Archaeology and Ethnology, Carnegie Institution of Washington, Department of Archaeology, vol. IV, No. 101, pp. 100-124. Cambridge, Massachusetts, 1951.

7. "Loop-Nose Incense Burners in the Guatemala National Museum", Notes on Middle American Archaeology and Ethnology, Carnegie Institution of Washington, Department of Archaeology, vol. IV, No. 103, pp. 143-158. Cambridge, Massachusetts, 1951.

8. "Further Notes on Three-Pronged Incense Burners and Rim-Head Vessels in Guatemala", Notes on Middle American Archaeology and Ethnology, Carnegie Institution of Washington, Department of Archaeology, vol. Iv, No. 105, pp. 162-176. Cambridge, Massachusetts, 1951.

9. "E1 Incensario de Guayasco Recientemente Donado al Museo Nacional de Arqueología y Etnología", Revista del Instituto de Antropología e Historia de Guatemala, vol. III, No. 2, pp. 41-44. Guatemala, 1951.

10. "Notes and Comments on 'Duck-pots' from Guatemala", Middle American Research Records, Middle American Research Institute, 
The Tulane University of Louisiana, vol. II, No. 1, publicación 17, pp. 1-16. New Orleans, 1952.

11. "El Juego de Pelota de los Mayas", Estudios, Revista de Cultura Hispánica, Duquesne University, año I, No. 4, pp. 29-32. Pittsburgh, 1952.

12. "Travertine Vase in the Guatemala National Museum", American Antiquity, Facts and Comments, vol. xvir, No. 3, pp. 254-256. Menasha, 1952.

13. “Arte Maya”, Horizonte, Publicación Mensual Ilustrada de Artes y Letras, año I, No. 2, junio, Guatemala, 1953.

14. "E1 Arte de Los Mayas", Estudios, Revista de Cultura Hispánica, Duquesne University, año II, No. 8, diciembre, pp. 23-27. Pittsburgh, Pa., 1953.

15. "A Brief Essay on the Development of Maya Art", El Palacio, vol. 61, No. 1, pp. 1-8. Santa Fe, 1954.

16. "Cultura Folk y Cultura Compleja en el Área Maya Meridional", Ciencias Sociales, Unión Panamericana, Departamento de Asuntos Culturales, vol. v, No. 26, pp. 50-63. Washington, 1954.

17. "Jointed Figurines in Mesoamerica and their Cultural Implication", Southwestern Journal of Antropology, The University of New Mexico Press, vol. 10, No. 3, Otoño, pp. 268-277. Albuquerque, 1954.

18. "Figurinas Articuladas de Mesoamérica", Revista del Instituto de Antropolơğía e Historia de Guatemala, vol. vi, No. 2, pp. 1-9. Guatemala, 1954.

19. "Comments on Incense Burners from Copan, Honduras", American Antiquity, Facts and Comments, vol. xx, No. 3, pp. 285-286. Menasha. Enero, 1955.

20. "Chinese Figurines in Mesoamerica", American Antiquity, Facts and Comments, vol. xx, No. 2, pp. 286-288. Menasha. Enero, 1955.

21. "Pottery Mask Traditions in Mesoamerica", Southwestern Journal of Anthropology, vol. II, No. 3, p. 205-213. Albuquerque, New Mexico, 1955.

22. "The Roots of Communism in Central America", The Oklahoma Quarterly, publicado por the University of Oklahoma Association, vol. IV, No. 4. Norman, Oklahoma, 1955.

23. "Pyramids in the Jungle (Tikal, Guatemala)", Oklahoma Anthropological Society Newsletter, vol. Iv, No. 7, pp. 2-6. Norman, Oklahoma, 1956.

24. "The Development of Folk and Complex Cultures in the Southern Maya Area", American Antiquity, vol. xxi, No. 4, pp. 343-356. Salt Lake City, Utah, 1956.

25. "The 1956 Summer Excavations in Guatemala", Oklahoma Anthropological Society Newsletter, vol. v, No. 3, pp. 8-10. Norman, Oklahoma, 1956.

26. "Summer Excavations in Guatemala", Archaeology, Archaeological Institute of America, vol. 9, No. 4, pp. 286-287. Brattleboro, Vermont, 1956. 
27. "E1 Incensario de 'Tres Asas' de Kaminaljuyú, Guatemala", Revista del Instituto de Antropología e Historia de Guatemala, vol. vIII, No. 2, pp. 3-8. Guatemala, 1956.

28. "Settlement Patterns in the Guatemalan Highlands: Past and Present", Pre-historic Settlement Patterns in the New World, editado por Gordon R. Willey, Viking Fund Publications in Anthropology, No. 23, pp. 101-106. New York, 1956.

29. "Incensario de Purulha, Guatemala", Revista del Instituto de Antropología e Historia de Guatemala, vol. Ix, No. 1, pp. 3-8. Guatemala City, 1957.

30. "Un Raro Cascabel de Barro del Periodo Primitivo Preclásico en Guatemala", Revista del Instituto de Antropología e Historia de Guatemala, vol. Ix, No. 1, pp. 1-12. Guatemala, 1957.

31. "Report upon Summer Activities in Guatemala and Mexico, 1957", Oklahoma Anthropological Society Newsletter, vol. vi, No. 3, pp. 4-7. Norman, Oklahoma, 1957.

32. "Evidence for Precolumbian Goiter in Guatemala", con Nevin S. Scrimshaw, American Antiquity, vol. 23, No. 2, parte 1, pp. 174-176. Utah, 1957.

33. "The Ball Game 1000 Years Ago", Sooner Magazine, publicado por University of Oklahoma Association, vol. 30, No. 3, pp. 5-23. Norman, Okla., 1957.

34. "Underwater Research in Guatemala", Archaeology, vol. 19, No. 4, pp. 282-283. Vermont, 1957.

35. "Aqualung Archaeology", Natural History Magazine, The Magazine of the American Museum of Natural History, vol. LXvir, No. 3, pp. 120-125. New York, 1957.

36. "From the Depths of Lake Amatitlan: Some of the Hundreds of Maya Antiquities Recently Found by Guatemalan Aqualung Divers", The Illustrated London Neres, vol. 233, No. 6214, pp. 70-72. Londres, 1958.

37. "Hallazgos Arqueológicos en Aguas del Lago de Amatitlán”, El Imparcial, 16 de agosto, pp. 13-16. Guatemala, 1958.

38. "Hallazgos Arqueológicos en Aguas del Lago de Amatitlán", Revista del Instituto de Antropología e Historia de Guatemala, vol. 10, No. 1, pp. 3-12. Guatemala, 1958.

39. "A Brief Essay on the Development of Maya Art", Bulletin of the Arts, No. 1, of the Serrer Museum. Shawnee, Oklahoma, 1958.

40. "Report on the 1958 Summer Activities in Guatemala", Oklahoma Anthropologica Society Newsletter, vol. viI, No. 3, pp. 7-10. Norman, 1958.

41. "Pêche au Trésor dans les Eaux Sacrées du Lac Amatitlan", Sciences et Avenir, No. 142, pp. 618-622.

42. "Figuras de Incensarios de tres Picos de la Colección 'Raúl Moreno', Guatemala”, Antropología e Historia de Guatemala, vol. x, No. 2, julio, 1958, pp. 13-15.

43. "Precolumbian Cultural Connections Between Mesoamerica and Ecuador", Middle American Research Records, vol. 2, No. 6, 1959, 
reimpreso de la publicación No. 18, pp. 141-156. Middle American Research Institute, Tulane University, New Orleans, 1959.

44. "Underwater Archaeology in the Maya Highlands", Scientific American, vol. 20, No. 3, pp. 100-113.

45. "Schatzsucher im Heiligan See Amatitlan", Lebendiges Wissen, Stuttgart. Febrero, 1959, pp. 59-63.

46. "Culto a la Imagen del Señor de Esquipulas en Centro América y Nuevo México", In IDAEH, vol. II, No. 1, Guatemala, pp. 44-49.

47. “The Composite or 'Assemble-it-Yourself' Censer: a New Lowland Maya Variety of the Three-Pronged Incense Burner", American Antiquity, vol. 25, No. 1, pp. 51-58. Salt Lake City (U. de Utah).

48. "Skin Diving: Report on Underwater Archaeology at Lake Amatitlan", en: Underwater Adventure, por Bill Barada (Trend Book 188), pp. 84-87. Los Ángeles, 1959.

49. "Pruebas de la Existencia de Bocio Precolombino en Guatemala", en: Oficina Sanitaria Panamericana, Boletin, Suplemento No. 3, pp. 157-161. Guatemala, enero, 1959.

50. “Cultura Folk y Cultura Compleja en el Área Maya Meridional”, Cuadernos del Seminario de Integración Social Guatemalteca, Primera Serie, No. 5, Guatemala, ed. por Jorge Luis Arriola, p. 31.

51. "America's Ball Game", Natural History, vol. 69, No. 1, pp. 48-59. New York, enero, 1960.

52. "Maya-Funde aus den Tiefen des Amatitlan-Sees", Die Umschau, vol. 13, pp. 402. Frankfurt, A. M., 1960.

53. "Research in Guatemala", Archaeology, vol. 13, No. 2, pp. 146-147. New York, 1960.

54. "Underwater Archaeology in Guatemala", Actas del Congreso Internacional de Americanistas, San José, Costa Rica, julio 20-27, 1958, vol. 2, pp. 229-240. San José, 1959.

55. "Middle America's Ball Game", Intercambio, órgano de la British Chamber of Commerce, No. 199, pp. 45-50, Pt. 1. México, junio 30, 1960.

56. "Middle America's Ball Game", Intercamóio, órgano de la British Chamber of Commerce, No. 200, pp. 41-45, Pt. 2. México, julio 31, 1960.

57. "Precolumbian Cultural Connections between Mesoamerica and Ecuador: Addenda", Middle American Research Records, vol. 2, No. 7, pp. 159-160. Middle American Research Institute, Tulane University, New Orleans, 1960.

58. "Amatitlan (Guatemala) Field Report", Katunob Neresletter Bulletin, vol. 1, No. 4, pp. 67-70, 1960.

59. "Pyramids in the Jungle; a Trip to the Ruins of Tikal, Guatemala, 1951”, Lore, vol. 11, No. 1, pp. 22-28. Milwaukee, 1960.

60. "Tesoro Maya en un Lago; los Buzos Hallan Antiquísimos Objetos", Life en Español, vol. 15, No. 9, pp. 23-27. Chicago, Illinois, mayo 16, 1960.

61. "Steps in Present-Day Pottery Making in Chinautla, Guatemala", Katunob Newsletter-Bulletin, vol. 2, No. 1, pp. 56-57, marzo, 1961. 
62. "Underwater Archaeological Studies in Lake Amatitlan, Highland Guatemala", American Philosophical Society Yearbook, pp. 547-551, 1960.

63. "Faces From a Maya Past", Life, pp. 77-80, Feb. 24, 1961.

64. "Miniature Mushroom Stones From Guatemala", American Antıquity, vol. 26, No. 4, pp. 498-504, abril, 1961.

65. "Shark Teeth, Stingray Spines, and Shark Fishing in Ancient Mexico and Central America", Southwestern Journal of Anthropology, vol. 17, No. 3, pp. 273-296, otoño, 1961.

66. "Ball Game Handstones and Ball Game Gloves", Essays in Precolumbian Art and Archaeology (por Samuel K. Lothrop y otros). Cambridge, Harvard University Press. Ensayo, No. 9, pp. 126-151.

67. "Pruebas de la existencia del Bocio Precolombino en Guatemala", (con Nevin S. Scrimshaw) Antropología e Historia de Guatemala, vol. 13 , No. 2 , pp. 21-26.

68. "The Hallucinogenic Mushrooms of Mexico and Psilocybin: A Bibliography" (with R. Gordon Wasson y otros), Botanical Museum Leaflets, Harvard University, vol. 20, No. 2. Sección de Arqueología, pp. 40-50. Cambridge, 1962.

69. "Precolumbian Pottery Mushrooms for Mesoamerica", American Antiquity, vol. 28, No. 3, pp. 328-338. Salt Lake City, University of Utah Press, 1963.

70. "Exploration in Lake Peten Itza", Archaeology, vol. 16, No. 1, pp. 14-24. New York, 1963.

71. "The Rubber Ball Game of Ancient America", Lore, vol. 13, No. 2, pp. 44-53. 1963. (También en Lore Leaflet, No. 8.)

72. "Excavations in Bilbao, Santa Lucía Cotzumalhuapa - Informe Preliminar" (con Lee A. Parsons, Peter Jensen y Robert Ritzenthaler) Antropología e Historia de Guatemala, vol. 15, No. 1, pp. 3-14, 1963.

73. "More Gifts to the Rain God", Lore, vol. 14, No. 1, pp. 16-21, 1963.

74. "Pares de Vasijas Gemelas Policromadas con Figuras Pintadas, del Ârea Maya", Estudios de Cultura Maya, vol. 3, pp. 107-112, 1963.

75. "Frozen in Clay", Lore, vol. 3, pp. 107-112, 1963.

76. "Precolumbian Ball Game Handstones: Rejoinder to Clune", American Antiquity, vol. 30, No 1, pp. 84-86, 1964.

77. "Precolumbian Cultural Similarities and Differences between the Highland Guatemalan and Tropical Rainforest Mayas", En el XXXV Congreso Internacional de Americanistas, pp. 215-224. México, Instituto Nacional de Antropología e Historia, 1964.

78. "Figurines Tell a Tale", Lore, vol. 14. No. 4, pp. 110-116, 1964.

79. "Enduring Villages of Western Mexico", Expedition, vol. 6 No. 4, pp. 28-33, 1964.

80. "Az Essöistennek szánt ajandékok a Chapala-tóbal", Elet es Tudomany, vol. 19. No. 37, p. 1769, 1964.

81. "Pares de Vasijas Gemelas Policromadas con Figuras Pintadas, del Ãrea Maya" (con Lee A. Pasons), Antropología e Historia de Guatemala, vol 16, No. 1, pp. 13-22, 5 ilus., 1964. 
82. "Ofrendas al Dios de la Lluvia", Antropología e Historia de Guatemala, vol. 16, N², pp. 38-48, 1964.

83. "Concerning Girard's reply to de Borhegyi's review of Los Mayas Eternos", American Antiquity, vol. 30, No. 4, pp. 498-499, 1965.

84. "The Art of the Ancient Maya", Lore, vol. 15, No. 1, pp. 18-28, 1964.

85. "Bilbao Site, Santa Lucía Cotzumalhuapa, Guatemala" (con Lee A. Parsons ), Katunob, vol. 15, No. 1, p. 25, 1965.

86. "Some Unusual Mesoamerican Portable Stone Sculpture in the Museum für Volkerkunde Berlin”, Baessler-Archiv, Neue Folge, Handbook of Middle American Indians, vol. 2, pp. 3-57-, 1965.

87. "The First Printers of the New World", Lore, vol. 16, No. 1, pp. $5-9,1965$.

88. "Archaelogical Synthesis of the Guatemalan Highlands", en The Handbook of Middle American Indians, vol. 2, pp. 3-58-, 1965.

89. "Settlement Patterns of the Guatemalan Highlands", en The Handbook of Middle American Indians, vol. 2, pp. 59-75, 1965.

90. "Precolumbian archaeological material in museums and private collections of southern Europe", en American Philosophical Association Yearbook, pp. 480-484.

91. “America's Oldest Dolls", Lore, vol. 16, No. 3, pp. 87-91, 1966.

92. "A Miniature Ceremonial Ballgame Yoke from Mexico", American Antiquity, xol. 31, No. 5, Pt. 1, pp. 742-4, 1966.

93. "The Development of Folk and Complex Cultures in the Southern Maya Area", Ancient Mesoamerica: Selected Readings, por John A. Graham, pp. 148-161. Palo Alto, California: Peek Publications, 1966.

94. “The Wind God's Breastplate", Expedition, vol. 8, No. 4, pp. 13-15, 1966.

95. "Shell Offerings and the Use of Shell Motifs at Lake Amatitlan, Guatemala, and Teotihuacan, Mexico", en Actas y Memorias del XXXVI Congreso Internacional de Americanistas, pp. 355-371. Sevilla, 1966.

96. "Eastern Chiapas (Chincultic report). American Antiquity, vol. 32, No. 1, "Current Research", p. 137.

97. "Underwater Archaeology in Guatemala", en Conquistadors without Swords: Archaelogists in the Americas, Ed. Leo Deuel. New York, St. Martin's Press., pp.371-380.

98. "Children of the Wind God", Pacific Discovery, vol. 20, No. 5, pp. 24-27.

99. "Miniature 'Thin Stone Heads' and Other Precolumbian Objects from Mesoamerica", American Antiquity, vol. 32, No. 4, pp. 543-546.

100. "Piedras Semiesféricas con Asas para el Juego de Pelota y 'Manoplas' en Mesoamérica: Una Posible Alternativa para su Función", Estudios de Cultura Maya, vol. 6, pp. 215-19. México, 1968.

101. "Una Fecha de C-14 para la Influencia Teotihuacana en Guatemala", Estudios de Cultura Maya, vol. 6, pp. 221-24. México, 1968. 
102. "Hoops, Yokes and Rubber Balls", Pacific Discovery, vol. 21, No. 3, pp. 10-15, 1968.

103. "Chinkultic in Chiapas, Mexico", Archaeology, vol. 21, No. 3, p. 212. 1968.

104. "Rolling Wheels", Lore, vol. 18, No. 4, pp. 110-116. 1968.

105. "Archaeological Reconnaissance of Chinkultic, Chiapas, Mexico", Middle American Research Institute, Publication, No. 26, 1968.

106. "The Wind God's Breastplate", Lore, vol. 19, No. 1, pp. 24-26, 1968.

107. "Potters of Guatemala", Pacific Discovery, vol. 21, No. 6, pp. 27-30, 1968.

108. "Una Hacha Tallada poco Frecuente Procedente de Kaminaljuyú", Antropología e Historia de Guatemala, vol. 19, No. 2, pp. 15-19, 1967.

109. "The Horse God of Peten Itza", Pacific Discovery, vol. 22, No. 4, pp. 27-32, 1969.

110. "Progress on the Preliminary Explorations of the Agua Azul Cenote, Chinkultic, Chiapas, Mexico by the Milwaukee Public Museum", Katunob, vol. 6, No. 4, pp. 20-22, 1969.

111. "Miniature and Small Stone Artifacts from Mesoamerica", Baessler-Archiv, N. F., Bd. 17, pp. 245-64, 1969.

112. "Stone, Bone, and Shell Objects from Lake Amatitlan, Guatemala", Baessler-Arvhic, N. F., Bd. 17, pp. 265-302, 1969.

113. In preparation: "The Ballgame in Mesoamerica: Its History and Implications", Proceedings of the XXXVIII International 'Congress of Americanists, Stuttgart and Munich, 1970.

114. "Wheels and Man", Archaelogy, vol. 23, No. 1, pp. 18-25. 